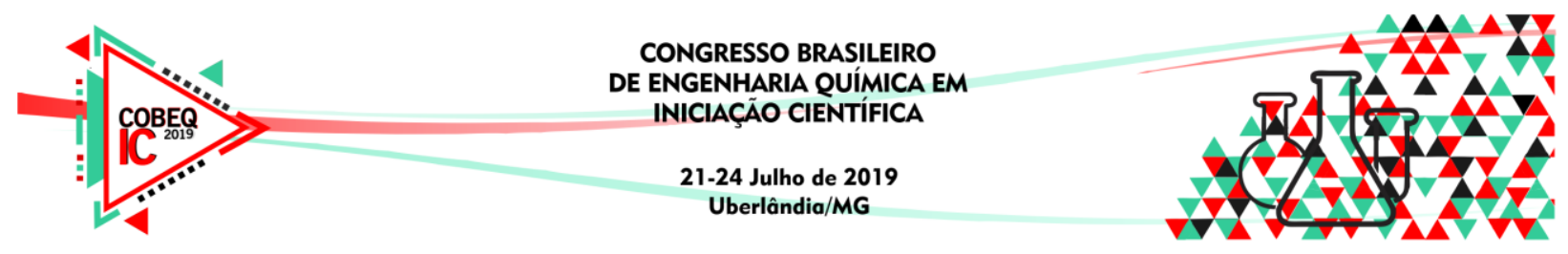

\title{
DETERMINAÇÃO DE PARÂMETROS DE FILTRAÇÃO EM MÓDULO DIDÁTICO FILTRO PRENSA - PROPOSTA DE DIMENSIONAMENTO A PARTIR DE ESCALA PILOTO
}

\author{
B. A. ROCHA ${ }^{1}$, G. P. ALVES ${ }^{1}$, I. F. NUNES ${ }^{1}$, I.B.C. AMARAL ${ }^{1}$ e A. B. REIS ${ }^{2}$ \\ ${ }^{1}$ Universidade Federal dos Vale do Jequitinhonha e Mucuri, Instituto de Ciência e Tecnologia \\ ${ }^{2}$ Universidade Federal dos Vales do Jequitinhonha e Mucuri, Departamento de Engenharia Química \\ Diamantina-MG, Brasil. \\ e-mail para contato: bruna.rocha1994@gmail.com
}

\begin{abstract}
RESUMO - A filtração é um método de separação mecânica de partículas por meio do uso de um meio filtrante, com objetivo de dissociar partículas sólidas presentes em um líquido. Entre os tipos de filtração, destacam-se o filtro a vácuo e o filtro prensa, que possuem diferentes escalas e aplicações. $O$ filtro a vácuo pode ser utilizado como uma escala piloto para determinar condições ótimas de operação em um filtro prensa, evitando assim, testes em grandes escalas. A filtração em escala piloto foi realizada utilizando $1 \mathrm{~L}$ da solução de carbonato de cálcio $\left(\mathrm{CaCO}_{3}\right)$ e uma amostra de jeans como meio filtrante para determinação de parâmetros como resistividade da torta e resistência do meio filtrante. A partir da teoria da filtração de tortas incompressíveis, foi possível estimar a relação entre o tempo e a área do meio filtrante e ajustar o tempo de filtração em um filtro prensa de volume igual a $20 \mathrm{~L}$ para as mesmas condições de concentração e meio filtrante. O tempo esperado para filtração da suspensão foi de 8 min e 47 s utilizando uma área de filtração equivalente a $0,16 \mathrm{~m}^{2}$, tendo sido, por fim, obtido o tempo total de filtração de 6 min e 23s. O resultado obtido encontra-se dentro do tempo esperado para a filtração, considerando que as diferenças operacionais do filtro prensa causam uma melhoria experimental em relação ao filtro a vácuo.
\end{abstract}

\section{INTRODUÇÃO}

A filtração é uma operação unitária que se baseia na separação de partículas sólidas suspensas de meios líquidos, na qual o fluido atravessa um meio filtrante, em virtude da diferença de pressão presente no meio, e o sólido se deposita no meio filtrante formando uma torta sólida (SILVA; FREITAS; SILVEIRA, 2012). Os processos de filtração são utilizados para produção de cerveja, vinhos e outros (UENOJO; PASTORE, 2007; SOUSA; COSTA; FOGAÇA, 2019), extração de corante (JOSÉ et al., 2019), tratamento de água (OTENIO et al., 2008) e outros. Fundamentalmente, a separação por filtração consiste na passagem de uma polpa por um meio poroso, subdividindo-se em filtrado e torta de filtração, sendo esta uma camada de sólida sobre o meio filtrante e o filtrado composto pela fase líquida restante no processo. Dessa forma, o objetivo da operação é separar mecanicamente as partículas sólidas 


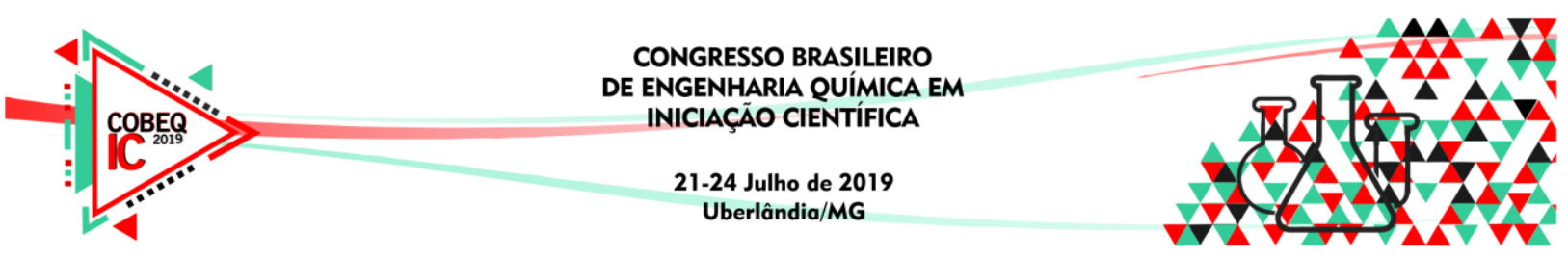

de uma suspensão líquida com o auxílio de um leito poroso (SILVA; FREITAS; SILVEIRA, 2012).

Dentre as técnicas de filtração, o filtro a vácuo e o filtro prensa são mais comumente utilizados. A filtração a vácuo é um processo acelerado, pois cria uma maior diferença de pressão nos dois lados do filtro, o que ocorre em virtude do aumento no fluxo do filtrado devido à sucção provocada pelo vácuo. Sua montagem consiste em um frasco Kitassato, provido de um funil de Büchner ligado a uma bomba de vácuo (SILVA; FREITAS; SILVEIRA, 2012). Por outro lado, os filtros do tipo prensa são equipamentos que apresentam um maior teor de sólidos secos nas tortas formadas com baixo consumo de energia, sendo necessária apenas uma bomba hidráulica para forçar a passagem da suspensão pelo equipamento. Sendo ainda possível o refluxo da suspensão, proporcionando melhor eficiência de filtração e consolidação das tortas. No filtro prensa são posicionadas várias placas justapostas e a filtração é realizada sob a ação de pressão exercida sobre a suspensão, havendo o bombeamento através dos compartimentos, obtendo-se a torta aderida aos meios filtrantes, que podem ser papéis de filtro, tecidos e/ou lonas (SILVA; FREITAS; SILVEIRA, 2012).

Os meios filtrantes considerados eficientes devem, segundo MacCabe et al. (1993): possuir boa retenção dos sólidos a serem filtrados sem impedir a passagem do fluido; fornecer um filtrado claro; possuir boa resistência química e física, sendo capaz de suportar as condições do processo; permitir que a torta seja retirada de forma limpa e completa; e ser de baixo custo.

No âmbito desse contexto, o presente trabalho teve como objetivo determinar o tempo de filtração a ser utilizado em módulo didático "filtro prensa", construído pela equipe para ser utilizado em aulas práticas. Para tanto, foram considerados dados obtidos a partir de experimentos realizados em sistema de filtração a vácuo e modelagem matemática.

\section{METODOLOGIA}

Os experimentos de filtração à vácuo foram realizados em escala de bancada com a utilização de funil de Büchner e frasco Kitassato acoplado a bomba peristáltica ajustada em 60 rpm alimentada com suspensão sob agitação constante, afim de se obterem dados experimentais para cálculo do tempo de filtração utilizado no módulo filtro prensa. Foi utilizado $1 \mathrm{~L}$ de suspensão aquosa de carbonato de cálcio $\left(\mathrm{CaCO}_{3}\right)$ na concentração de $20 \mathrm{~g} / \mathrm{L}$, obtida a partir da moagem em moinho de facas de giz comercial uniformizado em tamis 24 mesh. O meio filtrante utilizado foi o tecido jeans (comercial). O experimento permitiu coletar, a cada $100 \mathrm{~mL}$, dados referentes à queda de pressão da bomba, assim como o tempo necessário para toda a filtração.

Assim, a partir dos cálculos realizados na filtração a vácuo, foi possível obter a relação entre o tempo e a área do meio filtrante a fim de se estimar e ajustar o tempo total de filtração e calcular os parâmetros a serem reproduzidos no módulo didático filtro prensa. Para tanto foram preparados $20 \mathrm{~L}$ da mesma suspensão de $\mathrm{CaCO}_{3}$, mantida sob agitação constante, no tanque de alimentação. $\mathrm{O}$ experimento teve início com a abertura da válvula que permite a passagem da suspensão, em seguida, a bomba hidráulica foi acionada para que o fluido passasse pelo equipamento com a velocidade e pressão adequadas, possibilitando assim, a coleta de 


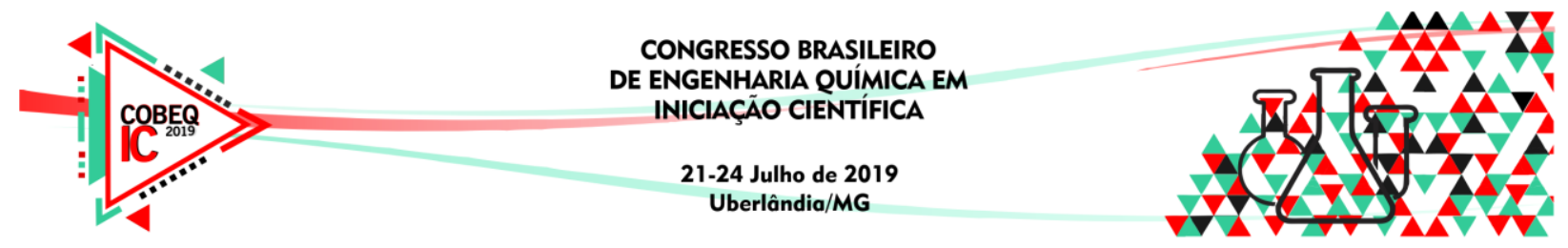

dados referentes à queda de pressão da bomba e também o tempo necessário para um ciclo de filtração.

O cálculo da resistividade da torta $(\alpha)$ é feito de acordo com a teoria da filtração para tortas incompressíveis de MacCabe et al. (1993), sendo afetada principalmente pelas propriedades da torta, como a porosidade e o diâmetro da partícula e pode ser definida pela Equação 1:

$$
\alpha=\frac{\Delta p_{c} A}{m_{c} u \mu}=\frac{K_{c} A^{2} \Delta p}{\mu C}
$$

Onde, $\Delta \mathrm{p}_{\mathrm{c}}$, queda de pressão na torta; $\mathrm{A}$, área de filtração; $\mathrm{m}_{\mathrm{c}}$, massa de sólidos na torta; $\mathrm{u}$, velocidade do filtrado; $\mu$, viscosidade do filtrado; $\Delta \mathrm{p}$, queda de pressão total e $\mathrm{C}$, concentração da suspensão.

Por sua vez, a resistência que o meio filtrante apresenta ao escoamento é influenciada pela queda de pressão, fazendo com que as partículas passem com maior velocidade pelo meio, aumentando a aderência de sólidos, conforme a Equação 2 (MACCABE et al., 1993):

$$
R_{m}=\frac{\Delta p_{m}}{u \mu}=\frac{1}{q_{0}} \frac{A \Delta p}{\mu}
$$

Onde, $\Delta \mathrm{p}_{\mathrm{m}}$, queda de pressão no meio filtrante e $\mathrm{q}_{0}$, vazão do filtrado.

Com a filtração à pressão constante, as únicas variáveis são o volume $(V)$ e o tempo $(t)$, logo, segundo MacCabe et al. (1993), tem-se:

$$
\frac{d t}{d V}=\frac{1}{q}=K_{c} V+\frac{1}{q_{0}}
$$

Integrando a Equação 3,

$$
\frac{t}{V}=\left(\frac{K_{c}}{2}\right) V+\frac{1}{q_{0}}
$$

A Equação 4 é considerada uma função linear $(t / V \times V)$ obtendo $K_{C}$ e $1 / q_{0}$ por meio dos coeficientes linear e angular da reta. Dessa forma, determinam-se os parâmetros: resistividade da torta $(\alpha)$ e resistência do meio filtrante $\left(R_{m}\right)$. O tempo de filtração pode ser determinado também a partir dos valores de $\alpha$ e $R_{m}$, conforme a Equação 5:

$$
t=\frac{\frac{\mu \alpha C}{A^{2} \Delta P}}{2} V^{2}+\frac{\mu R_{m}}{A \Delta P} V
$$




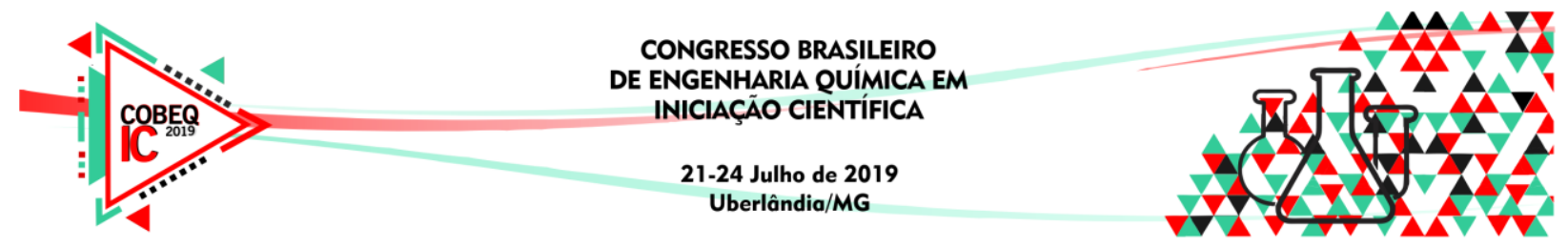

\section{RESULTADOS}

Na Figura 1 é apresentada a relação de $(t / V)$ em função de $V$, a partir da qual, com um ajuste linear a estes dado, obtêm-se os coeficientes linear $\left(1 / q_{0}\right)$ e angular $\left(K_{c}\right)$ da reta dada pela Equação 4.

Figura 1 - Relação de $t / V$ em função de $V$

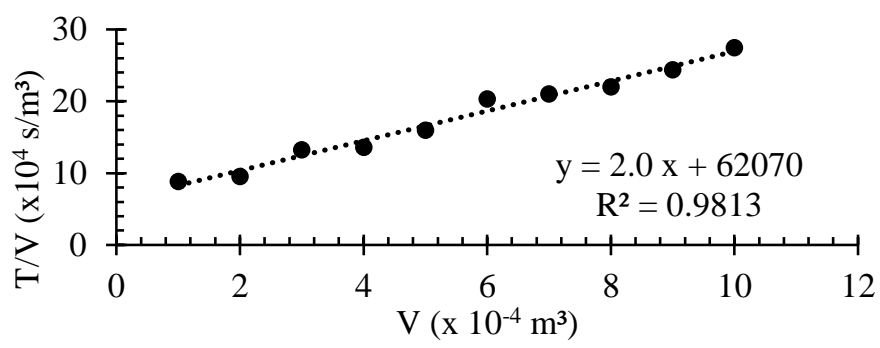

Os parâmetros necessários para calcular a resistividade da torta e a resistência do meio filtrante foram obtidas no ensaio de filtração a vácuo e encontram-se, em resumo, na Tabela 1.

Tabela 1 - Constantes de operação

\begin{tabular}{|c|c|c|}
\hline Constante & $\begin{array}{l}\text { Simbologia } \\
\text { (Unidade) }\end{array}$ & Valores obtidos \\
\hline Coeficiente angular & $K_{c}\left(s / m^{6}\right)$ & $2,00 \times 10^{8}$ \\
\hline Coeficiente linear & $1 / q_{0}\left(s / m^{3}\right)$ & 62070 \\
\hline Diferença de pressão & $\Delta p\left(N / m^{2}\right)$ & $3,73 \times 10^{4}$ \\
\hline Viscosidade do filtrado & $\mu(P a . s)$ & $8,86 \times 10^{-4}$ a $T=25^{\circ} \mathrm{C}($ MacCabe et al., 1993$)$ \\
\hline Área de filttração & $A\left(m^{2}\right)$ & $1,19 \times 10^{-2}$ \\
\hline Concentração da suspensão & $C\left(\mathrm{Kg} / \mathrm{m}^{3}\right)$ & 20 \\
\hline
\end{tabular}

Dessa forma, utilizando as Equações 1 e 2, de acordo com as constantes acima, obtiveramse os valores de $\alpha=1,19 \times 10^{11} \mathrm{~m} / \mathrm{kg}$ e $R_{m}=3,11 \times 10^{10} \mathrm{~m}^{-1}$.

Por fim, a partir dos parâmetros calculados, utilizou-se a Equação 5 para o cálculo da previsão do tempo de filtração no filtro prensa, mantendo os valores de $\alpha$ e $R_{m}$ constantes na filtração operada pelo filtro prensa, bem como a concentração da suspensão e a viscosidade do filtrado. $\mathrm{O}$ volume, a queda de pressão e a área, são parâmetros que se diferem da filtração a vácuo. $\mathrm{O}$ valor de $\Delta p$ utilizado para os cálculos foi equivalente ao valor obtido pela filtração à vácuo; sabe-se, porém, que se trata de uma estimativa, uma vez que a bomba utilizada no módulo filtro prensa trabalha com maiores pressões, portanto o tempo prático de filtração é menor do que o teórico.

Para determinar a melhor área de filtração, foi feita uma análise de como se comporta o tempo de filtração com o aumento da área, que pode ser operado a partir do aumento do número de placas no módulo. Cada placa possui uma área equivalente a $0,04 \mathrm{~m}^{2}$, desta forma, o cálculo do tempo foi feito utilizando com base na Equação 5, usando um volume de 20L, sendo obtida o tempo de filtração em função da área (Figura 2). 


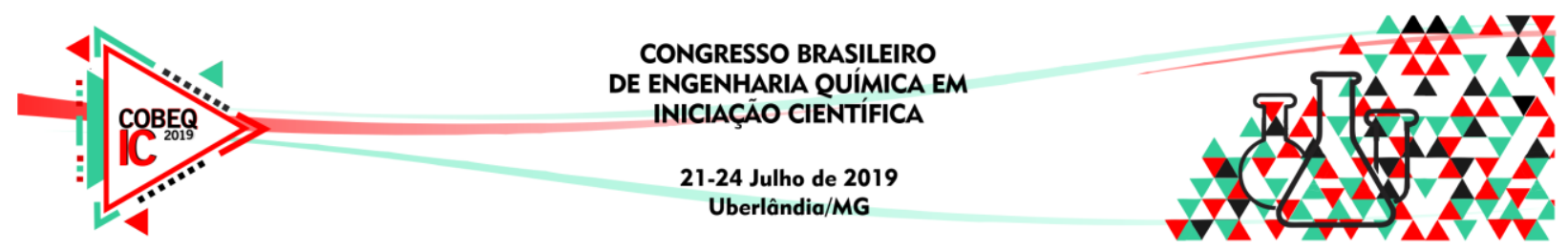

Figura 2 - Tempo versus Área

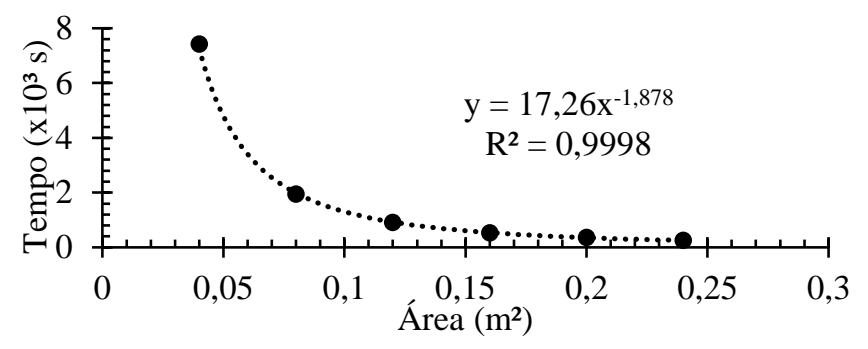

Dessa forma, verifica-se a relação exponencial entre a queda do tempo e o aumento da área, constatando-se que não é necessário utilizar mais de quatro unidades de área $\left(4 x 0,04 \mathrm{~m}^{2}\right)$, uma vez que não há queda expressiva no tempo de filtração. Logo, a área foi definida por $0,16 \mathrm{~m}^{2}$, com tempo teórico de filtração de $8 \mathrm{~min}$ e $47 \mathrm{~s}$. Ao fim do experimento com o filtro prensa, obteve-se o tempo de filtração de 6 min e $23 \mathrm{~s}$, devido à maior potência da bomba hidráulica, pois a queda de pressão registrada, de $5,88 \times 10^{4} \mathrm{~N} / \mathrm{m}^{2}$, foi superior à do ensaio com a bomba a vácuo. Assim, o tempo de filtração se encontra dentro do padrão esperados pelo experimento.

Por fim, a técnica de Microscopia Eletrônica de Varredura (MEV) possibilitou expor e comprovar a presença da torta de $\mathrm{CaCO}_{3}$ formada na filtração a vácuo em amplitudes e vistas distintas (Figura 3), e ainda, a superfície do meio filtrante (Figura 4) a qual demonstra a baixa porosidade do mesmo.

Figura 3 - Micrografias MEV das tortas de $\mathrm{CaCO}_{3}$

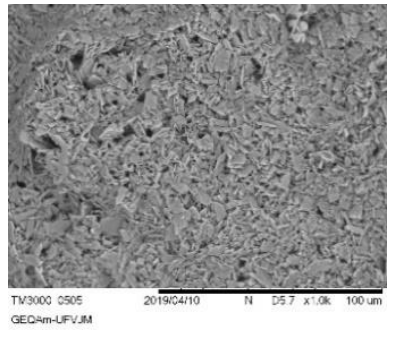

(a) - vista plana $1000 \mathrm{x}$

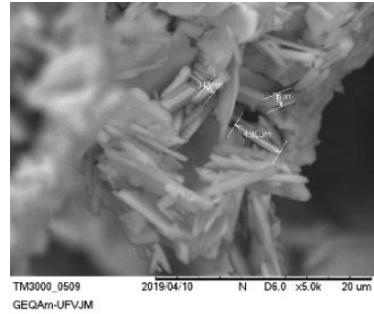

(b) - vista plana 5000x

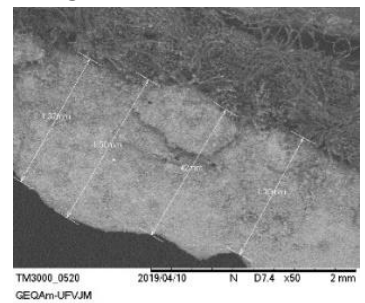

(c) - vista transversal 50x

Figura 4 - Micrografias MEV do meio filtrante

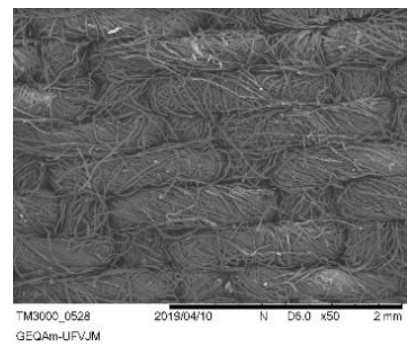

(a) - vista plana $50 \mathrm{x}$

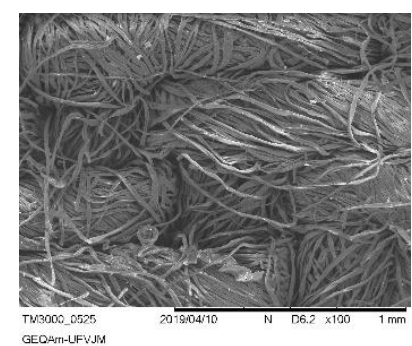

(b) - vista plana $100 \mathrm{x}$

A relação entre a baixa porosidade e a presença efetiva da torta é, de certa forma, intuitiva. Um meio filtrante composto por fibras bem estruturadas possui um baixo fator de porosidade, 


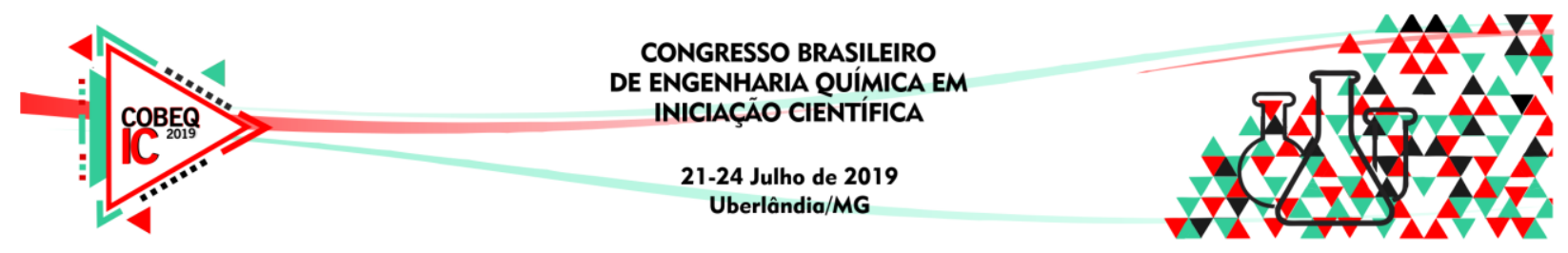

e como consequência, espera-se que retenha um maior número de partículas sólidas diante de um processo de filtração.

\section{CONCLUSÃO}

Foi possível verificar a eficácia da utilização da filtração à vácuo para determinação dos parâmetros a serem reproduzidos na filtração em módulo filtro prensa, uma vez que não se faz necessária a filtração de $20 \mathrm{~L}$ de solução para a determinação do $K_{c}$ e $\mathbf{1} / \boldsymbol{q}_{\mathbf{0}}$, parâmetros necessários pra se conhecer a resistividade da torta e a resistência do meio filtrante. Esses parâmetros foram calculados e foi possível ajustar o tempo de filtração de acordo com a área estipulada (quantidade de placas). Com isso, constatou-se que a área se comporta de maneira exponencial em relação ao tempo, visto que, à medida que se adicionam mais placas, o tempo de filtração será menor; porém, ao passo que se aumenta muito a unidade de área, o tempo de filtração tenderá a ser constante.

O experimento no filtro prensa comprovou que o tempo obtido comportou-se como esperado, tendo um tempo teórico equivalente a 8 min e $47 \mathrm{~s}$ para uma área de $0,16 \mathrm{~m}^{2}$ e um tempo experimental de 6 min e 23s. Para comparações em estudos posteriores, realizou-se a medição de sólidos em suspensão no filtrado para consolidar a eficiência da filtração no módulo didático construído.

\section{REFERÊNCIAS}

JOSÉ, E.; LOPES, D. S.; MACHADO, R. P.; GONZALES, F.; MUNDIM, L.; RODRIGUES, A. Padronização do corante natural extraído à partir do urucum (Bixa orellana) e sua aplicação na histologia. Collection Health, v. 11, n. 4, p. 1-12, 2019.

MCCABE, W. L.; SMITH, J. C.; HARRIOTT, P. Unit Operations Of Chemical Engineering. 5. Ed, p. 1-1154, 1993.

OTENIO, M. H.; PANCHONI, L. C.; CORREIA, G.; RAVANHANI, C.AVALIAÇÃO EM ESCALA LABORATORIAL DA UTILIZAÇÃO DO PROCESSO ELETROLÍTICO NO TRATAMENTO DE ÁGUAS. Química Nova, v. 31, n. 3, p. 508-513, 2008.

SILVA, M. R. DA; FREITAS, R. F.; SILVEIRA, R. B. DA. FILTRAÇÃO. p. 1-29, 2012.

SOUSA, V. M.; COSTA, L.; FOGAÇA, S. Perfil Físico-Químico de Cervejas Artesanais e Industriais e Adequação dos Rótulos Quanto à sua Graduação Alcoólica. Multidisciplinar e de Psicologia, v. 13, p. 440-447, 2019.

UENOJO, M.; PASTORE, G. M. Pectinases: Aplicações industriais e perspectivas. Quimica Nova, v. 30, n. 2, p. 388-394, 2007. 